\title{
Topological susceptibility in two flavors lattice QCD with exact chiral symmetry
}

\author{
Ting-Wai Chiu ${ }^{1,2,3}$, Tung-Han Hsieh ${ }^{* 4}$, Yao-Yuan Mao ${ }^{1}$ (TWQCD Collaboration) \\ ${ }^{1}$ Physics Department, National Taiwan University, Taipei 10617, Taiwan \\ ${ }^{2}$ Center for Quantum Science and Engineering, National Taiwan University, Taipei 10617, \\ Taiwan \\ ${ }^{3}$ Center for Theoretical Sciences, National Taiwan University, Taipei 10617, Taiwan \\ ${ }^{4}$ Research Center for Applied Sciences, Academia Sinica, Taipei 115, Taiwan
}

\begin{abstract}
We determine the topological susceptibility of the gauge configurations generated by lattice simulations using 2 flavors of optimal domain-wall fermion on the $16^{3} \times 32$ lattice with length 16 in the fifth dimension, at the inverse lattice spacing $a^{-1} \simeq 1.9 \mathrm{GeV}$. Using the adaptive thick-restart Lanczos algorithm, we project the low-lying eigenmodes of the overlap Dirac operator, and obtain the topological charge $Q_{t}$ of each gauge configuration in eight ensembles with pion masses in the range $230-580 \mathrm{MeV}$. From our result of $Q_{t}$, we compute the topological susceptibility and the second normalized cumulant. Our result of the topological susceptibility agrees with the sea-quark mass dependence predicted by the next-to-leading order chiral perturbation theory, and provides a determination of both the chiral condensate and the pion decay constant.
\end{abstract}

The XXIX International Symposium on Lattice Field Theory - Lattice 2011

July 10-16, 2011

Squaw Valley, Lake Tahoe, California

${ }^{*}$ Speaker. 


\section{Introduction}

The vacuum of Quantum Chromodynamics (QCD) has a non-trivial topological structure. The cluster property and the gauge invariance require that the ground state must be the $\theta$ vacuum, a superposition of gauge configurations in different topological sectors. The topological susceptibility $\left(\chi_{t}\right)$ is the most crucial quantity to measure the topological fluctuations of the QCD vacuum, which plays an important role in breaking the $U_{A}(1)$ symmetry. Theoretically, $\chi_{t}$ is defined as

$$
\chi_{t}=\int d^{4} x\langle\rho(x) \rho(0)\rangle, \quad \rho(x)=\frac{1}{32 \pi^{2}} \varepsilon_{\mu v \lambda \sigma} \operatorname{tr}\left[F_{\mu v}(x) F_{\lambda \sigma}(x)\right],
$$

where $\rho(x)$ is the topological charge density expressed in term of the matrix-valued field tensor $F_{\mu \nu}$. With mild assumptions, Witten [1] and Veneziano [2] obtained a relationship between the topological susceptibility in the quenched approximation and the mass of $\eta^{\prime}$ meson (flavor singlet) in the full QCD. This implies that the mass of $\eta^{\prime}$ is essentially due to the axial anomaly relating to non-trivial topological fluctuations, unlike those of the (non-singlet) approximate Goldstone bosons.

From (1.1), we obtain

$$
\chi_{t}=\frac{\left\langle Q_{t}^{2}\right\rangle}{\Omega}, \quad Q_{t} \equiv \int d^{4} x \rho(x),
$$

where $\Omega$ is the volume of the system, and $Q_{t}$ is the topological charge (which is an integer for QCD). Thus, one can determine $\chi_{t}$ by counting the number of gauge configurations for each topological sector. Furthermore, we can also obtain the second normalized cumulant

$$
c_{4}=-\frac{1}{\Omega}\left[\left\langle Q_{t}^{4}\right\rangle-3\left\langle Q_{t}^{2}\right\rangle^{2}\right],
$$

which is related to the leading anomalous contribution to the $\eta^{\prime}-\eta^{\prime}$ scattering amplitude in QCD, as well as the dependence of the vacuum energy on the vacuum angle $\theta$.

However, for lattice $\mathrm{QCD}$, it is difficult to extract $\rho(x)$ and $Q_{t}$ unambiguously from the gauge link variables, due to their rather strong fluctuations. To circumvent this difficulty, we may consider the Atiyah-Singer index theorem [3], $Q_{t}=n_{+}-n_{-}=\operatorname{index}(\mathscr{D})$, where $n_{ \pm}$is the number of zero modes of the massless Dirac operator $\mathscr{D} \equiv \gamma_{\mu}\left(\partial_{\mu}+i g A_{\mu}\right)$ with \pm chirality.

For lattice QCD with exact chiral symmetry, it is well-known that the overlap Dirac operator $[4,5]$ in a topologically non-trivial gauge background possesses exact zero modes (with definite chirality) satisfying the Atiyah-Singer index theorem. Thus we can obtain the topological charge from the index of the overlap Dirac operator. Writing the overlap Dirac operator as

$$
D_{o}=m_{0}\left(1+\gamma_{5} \frac{H_{w}}{\sqrt{H_{w}^{2}}}\right),
$$

where $H_{w}$ is the standard Hermitian Wilson operator with negative mass $-m_{0}\left(0<m_{0}<2\right)$, then its index is

$$
\operatorname{index}(D)=\operatorname{Tr}\left[\gamma_{5}\left(1-\frac{D_{o}}{2 m_{0}}\right)\right]=n_{+}-n_{-}=Q_{t}
$$


where $\operatorname{Tr}$ denotes trace over Dirac, color, and site indices.

In Ref [6], we measure the topological charge of the gauge configurations generated by lattice simulations of two flavors QCD on a $16^{3} \times 32$ lattice, with the optimal domain-wall fermion (ODWF) [7] at $N_{s}=16$, and plaquette gauge action at $\beta=5.95$, for eight sea-quark masses $m_{q} a=0.01, \cdots, 0.08$ with the interval 0.01 .

Mathematically, ODWF is a theoretical framework which can preserve the chiral symmetry optimally for any given $N_{s}$, with a set of analytical weights $\left\{\omega_{s}, s=1, \cdots, N_{s}\right\}$, one for each layer in the fifth dimension [7]. Thus the artifacts due to the chiral symmetry breaking with finite $N_{s}$ can be reduced to the minimum, especially in the chiral regime. The 4-dimensional effective Dirac operator of massless ODWF is

$$
D=m_{0}\left[1+\gamma_{5} S_{\text {opt }}\left(H_{w}\right)\right], \quad S_{\text {opt }}\left(H_{w}\right)=\frac{1-\prod_{s=1}^{N_{s}} T_{s}}{1+\prod_{s=1}^{N_{s}} T_{s}}, \quad T_{s}=\frac{1-\omega_{s} H_{w}}{1+\omega_{s} H_{w}},
$$

which is exactly equal to the Zolotarev optimal rational approximation of the overlap Dirac operator. That is, $S_{\text {opt }}\left(H_{w}\right)=H_{w} R_{Z}\left(H_{w}\right)$, where $R_{Z}\left(H_{w}\right)$ is the optimal rational approximation of $\left(H_{w}^{2}\right)^{-1 / 2}[8,9]$.

We use the adaptive thick-restart Lanczos algorithm [10] to project the low-lying eigenmodes of the 4-dimensional effective Dirac operator (1.4), and obtain the topological charge $Q_{t}$ of each gauge configuration. Then we compute the topological susceptibilty $\chi_{t}$ and the second normalized cumulant $c_{4}$, and compare our results to the Chiral Perturbation Theory (ChPT). We summarize the ChPT formulas as follows.

In 1992, Leutwyler and Smilga [11] derived the relationship between $\chi_{t}$ and the quark mass, at the leading order in ChPT. For 2 flavors QCD, it reads

$$
\chi_{t}=\Sigma\left(m_{u}^{-1}+m_{d}^{-1}\right)^{-1}
$$

where $m_{u}, m_{d}$ are the quark masses, and $\Sigma$ is the chiral condensate. This implies that in the chiral limit $\left(m_{u} \rightarrow 0\right)$ the topological susceptibility is suppressed by the internal quark loops. Most importantly, (1.5) provides a viable way to extract $\Sigma$ from $\chi_{t}$ in the chiral regime.

Recently, the topological susceptibility has been derived to the one-loop order in ChPT for an arbitrary number of flavors [12]. For $N_{f}=2$ with degenerate $u$ and $d$ quark masses $\left(m_{u}=m_{d} \equiv m_{q}\right)$, the foumula reduces to

$$
\frac{\chi_{t}}{m_{q}}=\frac{\Sigma}{2}\left\{1-3\left(\frac{\Sigma m_{q}}{16 \pi^{2} F_{\pi}^{4}}\right) \ln \left(\frac{2 \Sigma m_{q}}{F_{\pi}^{2} \mu_{s u b}^{2}}\right)+32\left(\frac{\Sigma}{F_{\pi}^{4}}\right)\left(2 L_{6}+2 L_{7}+L_{8}\right) m_{q}\right\},
$$

where $L_{i}$ are renormalized low-energy coupling constants defined at $\mu_{s u b}$ [13]. In this work, we fix $\mu_{\text {sub }}=770 \mathrm{MeV}$.

In this proceeding, we review our result of topological susceptibility presented in Ref. [6].

\section{Lattice Setup}

Simulations are carried out for two flavors QCD on a $16^{3} \times 32$ lattice at the lattice spacing $a \sim 0.1 \mathrm{fm}$, for eight sea-quark masses $m_{q} a=0.01,0.02,0.03,0.04,0.05,0.06,0.07$, and 0.08 , respectively. For the quark part, we use the optimal domain-wall fermion with $N_{s}=16$. For the 
gluon part, we use the plaquette action at $\beta=5$.95. An outline of our simulation algorithm and its acceleration with Nvidia GPUs have been presented in Refs. [14, 15].

For each sea-quark mass, we perform hybrid Monte-Carlo simulations on 30 GPUs independently, with each GPU generating 400 trajectories. After discarding 300 trajectories for thermalization, each GPU yields 100 trajectories. Thus, with 30 GPUs running independently, we accumulated total 3000 trajectories for each sea-quark mass. From the saturation of the binning error of the plaquette, as well as the evolution of the topological charge, we estimate the autocorrelation time to be around 10 trajectories. Thus we sample one configuration every 10 trajectories, then we have 300 configurations for each sea-quark mass. With a GPU cluster of 250 GPUs, we can simulate 8 sea-quark masses concurrently. It takes about 5 months to complete the simulations for the $\beta=5.95$ ensemble.

We determine the lattice spacing by heavy quark potential with Sommer parameter $r_{0}=$ $0.49 \mathrm{fm}$. Using the linear fit, we obtain the lattice spacing in the chiral limit, $a=0.1032(2) \mathrm{fm}$, which gives $a^{-1}=1.911(4)(6) \mathrm{GeV}$, where the systematic error is estimated with the uncertainty of $r_{0}$.

For each configuration, we calculate the zero modes plus 80 conjugate pairs of the lowest-lying eignmodes of the overlap Dirac operator. We outline our procedures as follows. First, we project 240 low-lying eigenmodes of $H_{w}^{2}$ using adaptive thick-restart Lanczos alogorithm ( $a$-TRLan) [10], where each eigenmode has a residual less than $10^{-12}$. Then we approximate the sign function of the overlap operator by the Zolotarev optimal rational approximation with 64 poles, where the coefficents are fixed with $\lambda_{\max }^{2}=(6.4)^{2}$, and $\lambda_{\min }^{2}$ equal to the maximum of the 240 projected eigenvalues of $H_{w}^{2}$. Then the sign function error is less than $10^{-14}$. Using the 240 low-modes of $H_{w}^{2}$ and the Zolotarev approximation with 64 poles, we use the $a$-TRLan algorithm again to project the zero modes plus 80 conjugate pairs of the lowest-lying eignmodes of the overlap operator, where each eigenmode has a residual less than $10^{-12}$. We store all projected eigenmodes for the later use. In this work, we use the index of the zero modes to compute $\chi_{t}$ and $c_{4}$.

\section{Results}

In Fig. 1, we plot the histogram of topological charge distribution for $m_{q} a=0.01,0.02, \cdots$, 0.08 respectively. Evidently, the probability distribution of $Q_{t}$ for each sea-quark mass behaves like a Gaussian, and it becomes more sharply peaked around $Q_{t}=0$ as the sea-quark mass $m_{q}$ gets smaller.

Using the result of $Q_{t}$, we compute the topological susceptibility $\chi_{t}$ (1.2), and the second normalized cumulant $c_{4}$ (1.3). In Table 1 , we list our results of $\chi_{t}, c_{4}$, and the ratio $c_{4} / \chi_{t}$. The error is estimated using the jackknife method with bin size of 10 configurations, with which the statistical error saturates.

Evidently, the statistical error of the topological susceptibility is about $10 \%$, while that of $c_{4}$ is very large due to low statistics. Therefore, we cannot draw any conclusions from our result of $c_{4}$, as well as from the ratio $c_{4} / \chi_{t}$.

In Fig. 2-(a), we plot our data of $\chi_{t}$ versus the sea quark mass $m_{q}$. The data points of $\chi_{t}$ are well fitted by the Leutwyler and Smilga formula (1.5) with $\Sigma a^{3}=0.00200(15)$. The fitted curve is plotted as the solid line in Fig. 2-(a). 

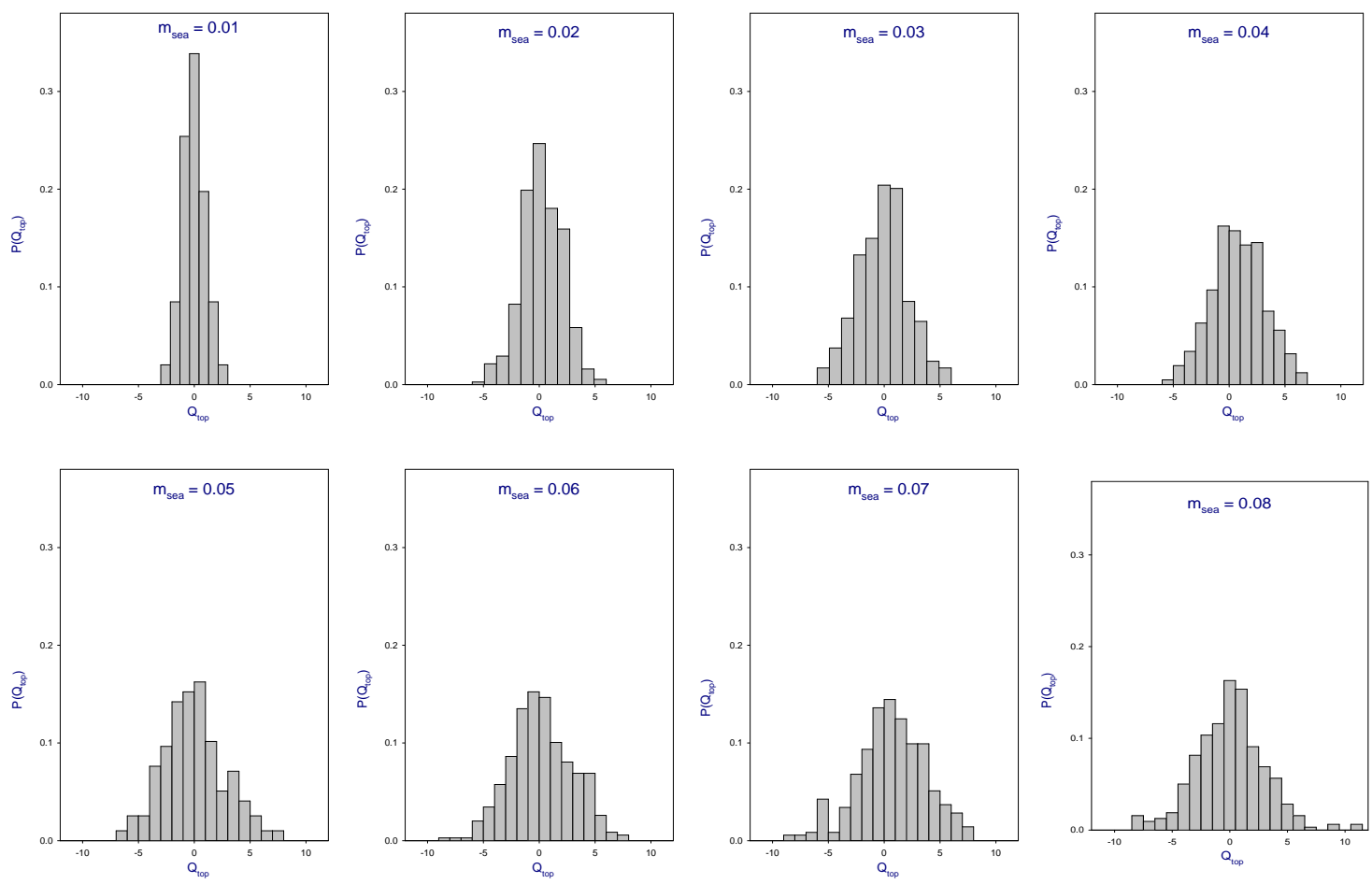

Figure 1: Histogram of topological charge distribution for eight sea quark masses, $m_{q} a=0.01,0.02,0.03$, $0.04,0.05,0.06,0.07$, and 0.08 respectively.

In Fig. 2-(b), we plot our data of $\chi_{t} / m_{q}$ versus the sea-quark mass $m_{q}$. The data points of $\chi_{t} / m_{q}$ are well fitted by the NLO ChPT formula (1.6) with $\Sigma a^{3}=0.0020(2), F_{\pi} a=0.048(7)$, and $\left(2 L_{6}+2 L_{7}+L_{8}\right)=-0.0001(3)$, where $\mu_{\text {sub }}=770 \mathrm{MeV}$. Using $a^{-1}=1.911(4)(6) \mathrm{GeV}$, we obtain $\Sigma=[241(6)(1) \mathrm{MeV}]^{3}$, and

$$
F_{\pi}=92(12)(2) \mathrm{MeV},
$$

where the errors represent a combined statistical error and the systematic error respectively.

In order to convert $\Sigma$ to that in the $\overline{\mathrm{MS}}$ scheme, we calculate the renormalization factor $Z_{s}^{\overline{\mathrm{MS}}}(2 \mathrm{GeV})$ using the non-perturbative renormalization technique through the RI/MOM scheme [16], and our result is [17]

$$
Z_{s}^{\overline{\mathrm{MS}}}(2 \mathrm{GeV})=1.244(18)(39) .
$$

Then the value of $\Sigma$ is transcribed to

$$
\Sigma^{\overline{\mathrm{MS}}}(2 \mathrm{GeV})=[259(6)(7) \mathrm{MeV}]^{3},
$$

where the errors represent a combined statistical error $\left(a^{-1}\right.$ and $\left.Z_{s}^{\overline{\mathrm{MS}}}\right)$ and the systematic error respectively. Since the present calculation is done at a single lattice spacing, the discretization error cannot be quantified reliably, but we do not expect much larger error because the optimal domain-wall fermion action is free from $O(a)$ discretization effects. Our result of $\Sigma(3.2)$ is in good 


\begin{tabular}{|c|ccc|}
\hline$m_{q} a$ & $\chi_{t}$ & $c_{4}$ & $c_{4} / \chi_{t}$ \\
\hline 0.01 & $1.13(10) \times 10^{-5}$ & $-1.22(1.15) \times 10^{-5}$ & $-1.07(1.01)$ \\
0.02 & $2.24(18) \times 10^{-5}$ & $-3.79(2.77) \times 10^{-5}$ & $-1.69(1.24)$ \\
0.03 & $3.29(27) \times 10^{-5}$ & $-1.25(2.87) \times 10^{-5}$ & $-0.04(0.87)$ \\
0.04 & $4.40(30) \times 10^{-5}$ & $6.39(4.16) \times 10^{-5}$ & $1.45(95)$ \\
0.05 & $5.31(41) \times 10^{-5}$ & $5.75(7.96) \times 10^{-5}$ & $1.08(1.50)$ \\
0.06 & $6.04(44) \times 10^{-5}$ & $1.00(1.09) \times 10^{-4}$ & $1.66(1.81)$ \\
0.07 & $7.24(55) \times 10^{-5}$ & $-5.12(134) \times 10^{-6}$ & $-0.07(1.86)$ \\
0.08 & $7.01(79) \times 10^{-5}$ & $-7.10(6.58) \times 10^{-4}$ & $-10.13(9.46)$ \\
\hline
\end{tabular}

Table 1: The topological susceptibility $\chi_{t}$, the second normalized cumulant $c_{4}$, and their ratio $c_{4} / \chi_{t}$, versus the sea quark masses, for $N_{f}=2$ lattice QCD with the optimal domain-wall fermion.

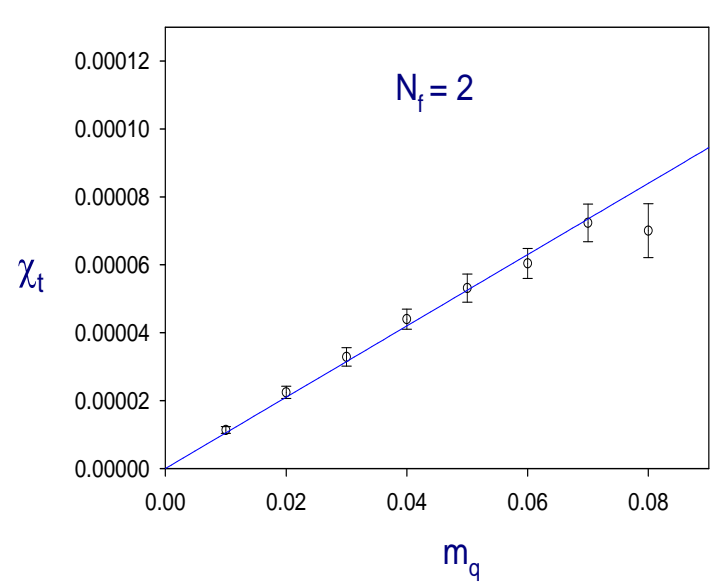

(a)

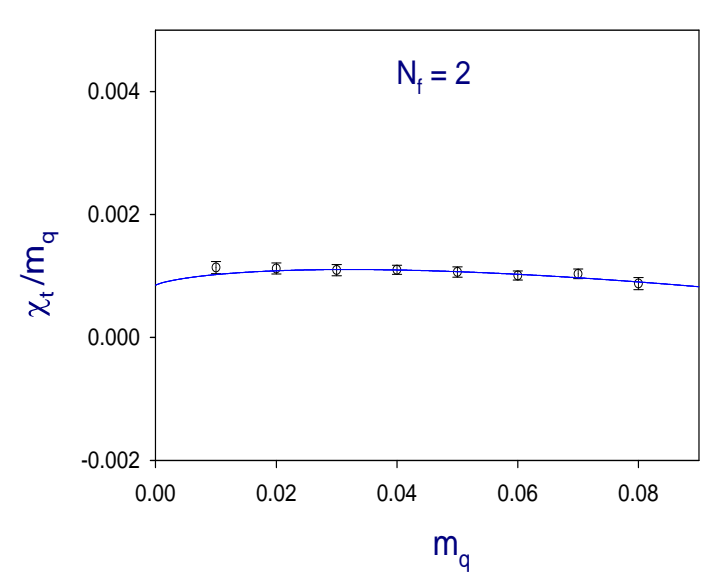

(b)

Figure 2: (a) The topological susceptibility $\chi_{t}$, and (b) the ratio $\chi_{t} / m_{q}$, versus the sea-quark mass $m_{q}$, for 2 flavors lattice QCD with ODWF.

agreement with that extracted from $\chi_{t}$ in $(2+1)$ flavors QCD with domain-wall fermion [18], as well as with those extracted from $\chi_{t}$ in $N_{f}=2$ and $N_{f}=2+1$ lattice QCD with overlap fermion in a fixed topology $[19,20]$.

\section{Concluding remark}

To summarize, we measure the topological charge of the gauge configurations generated by lattice simulations of 2 flavors QCD with the optimal domain-wall fermion at $N_{s}=16$ and plaquette gauge action at $\beta=5.95$, on a $16^{3} \times 32$ lattice. We use the adaptive thick-restart Lanczos algorithm to compute the low-lying eigenmodes of the overlap Dirac operator, and obtain the topological charge of each gauge configuration, and from which we compute the topological susceptibility for 8 sea-quark masses, each of 300 configurations. Our result of the topological susceptibility agrees with the sea-quark mass dependence predicted by the NLO ChPT formula (1.6), and gives the first 
determination of both the pion decay constant (3.1) and the chiral condensate (3.2) simultaneously from the topological susceptibility.

Recently, we have also computed the mass and decay constant of the pseudoscalar meson for the same set of gauge configurations [21]. Our data is in good agreement with the sea-quark mass dependence predicted by the next-to-leading order (NLO) ChPT, and provides a determination of the low-energy constants $\bar{l}_{3}$ and $\bar{l}_{4}$, the pion decay constant, the chiral condensate, and the average up and down quark mass. In view of our results in Refs. [6,21], we are confident that the nonperturbative chiral dynamics of the sea quarks are well under control in our HMC simulations. Moreover, this also implies that one can perform a large-scale simulation of unquenched lattice QCD with ODWF, which not only preserves the chiral symmetry to a high precision, but also samples all topological sectors ergodically.

This work is supported in part by the National Science Council (Nos. NSC99-2112-M-002012-MY3, NSC99-2112-M-001-014-MY3) and NTU-CQSE (No. 10R80914-4). We also thank NCHC and NTU-CC for providing facilities to perform part of our calculations.

\section{References}

[1] E. Witten, Nucl. Phys. B 156, 269 (1979).

[2] G. Veneziano, Nucl. Phys. B 159, 213 (1979).

[3] M. F. Atiyah and I. M. Singer, Annals Math. 87, 484 (1968).

[4] H. Neuberger, Phys. Lett. B 417, 141 (1998)

[5] R. Narayanan and H. Neuberger, Nucl. Phys. B 443, 305 (1995)

[6] T. W. Chiu, T. H. Hsieh and Y. Y. Mao [TWQCD Collaboration], Phys. Lett. B 702, 131 (2011).

[7] T. W. Chiu, Phys. Rev. Lett. 90, 071601 (2003); Nucl. Phys. Proc. Suppl. 129, 135 (2004)

[8] N. I. Akhiezer, "Theory of approximation", Dover, New York, 1992.

[9] T. W. Chiu, T. H. Hsieh, C. H. Huang and T. R. Huang, Phys. Rev. D 66, 114502 (2002)

[10] I. Yamazaki, Z. Bai, H. Simon, L.W. Wang, and K. Wu, ACM Transactions on Mathematical Software, Vol. 37, No. 3, Article 27 (2010).

[11] H. Leutwyler and A. Smilga, Phys. Rev. D 46, 5607 (1992).

[12] Y. Y. Mao and T. W. Chiu [TWQCD Collaboration], Phys. Rev. D 80, 034502 (2009)

[13] J. Gasser and H. Leutwyler, Nucl. Phys. B 250, 465 (1985).

[14] T. W. Chiu et al. [TWQCD Collaboration], PoS LATTICE2009, 034 (2009)

[15] T. W. Chiu et al. [TWQCD Collaboration], PoS LATTICE2010, 030 (2010).

[16] G. Martinelli, C. Pittori, C. T. Sachrajda, M. Testa and A. Vladikas, Nucl. Phys. B 445, 81 (1995)

[17] T. W. Chiu et al. [TWQCD Collaboration], "Nonperturbative renormalization of bilinear operators in lattice QCD with the optimal domain-wall fermion", in preparation.

[18] T. W. Chiu, T. H. Hsieh and P. K. Tseng [TWQCD Collaboration], Phys. Lett. B 671, 135 (2009)

[19] S. Aoki et al. [JLQCD and TWQCD Collaborations], Phys. Lett. B 665, 294 (2008)

[20] T. W. Chiu et al. [JLQCD and TWQCD Collaborations], PoS LATTICE2008, 072 (2008).

[21] T. W. Chiu, T. H. Hsieh and Y. Y. Mao [TWQCD Collaboration], arXiv:1109.3675 [hep-lat]. 